\title{
Reviewers for volumes 15, 16 and 17.1
}

The editors would like to thank the following reviewers for their contribution to volumes 15, 16 and 17.1 of $A L T-J$ :

\author{
Alsop, Graham \\ Anderson, Terry \\ Arbaugh, J. Ben \\ Aspin, Rob \\ Avery, Nicola \\ Baker, Jason \\ Bates, Reid \\ Bayne, Sian \\ Beetham, Helen \\ Bell, Frances \\ Benfield, Greg \\ Boyle, Tom \\ Brittain, Sandy \\ Brown, Stephen \\ Buckner, Kathy \\ Campbell, Lorna \\ Carey, Thomas \\ Collis, Betty \\ Connolly, Thomas \\ Conole, Grainne \\ Cook, John \\ Cook, Julian \\ Cooke, Louise \\ Cornelius, Sarah \\ Cousin, Glynis \\ Crook, Charles \\ Culver, Juliette \\ Daly, Caroline \\ de Freitas, Sara \\ de Laat, Maarten \\ Deepwell, Frances \\ Dempster, Jay \\ Doherty, Catherine \\ Dyke, Martin \\ Ellis, Robert \\ Fill, Karen
}

Fox, Bob

Gibbs, Graham

Gillam, Lee

Goodyear, Peter

Grabinger, Scot

Gunn, Cathy

Hanson, Janet

Hartley, Roger

Harvey, Jen

Hatzipanogos, Stylianos

Hedberg, John

Herodota, Thea

Hockings, Chris

Holley, Debbie

Hudson, Allison

Hughes, Gwyneth

Ingraham, Bruce

Jameson, Jill

Jara, Magdalena

Jefferies, Amanda

Jewitt, Carey

Jones, Ann

Jones, Chris

Kear, Karen

Keegan, Helen

Kennedy, Gregor

Keppell, Mike

Kiili, Kristian

Kirkwood, Adrian

Kraan, Wilbert

Kukulska Hulme, Agnes

Land, Ray

Levy, Philippa

Lisewski, Bernard

Littlejohn, Allison

Livingstone, Daniel
MacKeogh, Kay

MacLeod, Hamish

Maharg, Paul

Masiello, Italo

McKenna, Colleen

McNaught, Carmel

McSporran, Mae

Minocha, Shailey

Mogey, Nora

Morón-García, Sue

Naidu, Som

Newland, Barbara

Nicol, David

O’Donoghue, John

Oliver, Martin

Oliver, Ron

O'Rourke, Rebecca

Pachler, Norbert

Panda, Santosh

Pelletier, Caroline

Phillips, Rob

Preece, Jenny

Price, Sara

Ramanau, Ruslan

Ravenscroft, Andrew

Richardson, John

Riddy, Paul

Rieber, Lloyd

Roberts, George

Ryan, Malcolm

Sanders, John

Saunders, Gunther

Schmoller, Seb

Selinger, Michelle

Sharpe, Rhona

Sharples, Mike 
Simpson, Vicki

Sinclair, Christine

Sloep, Peter

Smith, Christine

Smith, Janice

Squire, Kurt

Stansfield, Mark

Stiles, Mark

Thompsett, Chris
Timmis, Sue

Torris-Steele, Geraldine

Traxler, John

Twining, Peter

Valcke, Martin

Van Harmelen, Mark

Ward, Robert

Warren, Adam

Weller, Martin
Weston, Pippa

Wheeler, Steve

White, Sue

Whitehouse, Christine

Whitelock, Denise

Whitton, Nicola

Wills, Sandra

Wilson, Scott

As editors, our approach continues to be one of supporting authors in developing and improving their submissions to $A L T-J$ through a constructive, iterative process. Our reviewers enable us to do this by providing detailed and considered feedback to authors. Collectively our reviewers have contributed to enhancing the quality of $A L T-J$, and we are extremely grateful.

This period has seen the transition from our previous submission system to Manuscript Central; this has made compiling a comprehensive list of reviewers challenging, as a consequence we may have missed some, for which we apologise.

Frances Bell and Rhona Sharpe Editors, ALT-J 